\title{
Comments on Letter to the Editor "Unilateral intravitreal dexamethazone implant for bilateral retinitis pigmentosa-related macular edema"
}

\author{
Giuseppe Querques • Mayer Srour • Eric H. Souied
}

Received: 6 August 2013 / Accepted: 8 August 2013 / Published online: 31 August 2013

(C) Springer-Verlag Berlin Heidelberg 2013

Dear Editor,

We thank Drs Alhassan and Quintyn for their interest in our recent article [1], and for sharing their observation of bilateral reduction of macular edema (ME) secondary to retinitis pigmentosa (RP) after unilateral intravitreal dexamethazone implant administration.

Intravitreal dexamethasone implant has recently become available for ophthalmology use, with the indication of ME secondary to retinal vein occlusion (RVO). However, as correctly pointed out by the authors, in RP, autoimmune process [2] and sustained chronic inflammatory reaction [3] may be involved in the development of ME, which explains why intravitreal steroids, and specifically intravitreal dexamethazone implant, might be considered as a valuable treatment option.

The authors reported morphological (macular thickness) and functional (visual acuity) improvement in the fellow eye of a 22-year-old RP patient with bilateral ME, as soon as 1 week after unilateral intravitreal dexamethazone implant administration. It is noteworthy that this patient had been receiving carbonic anhydrase inhibitors and brinzolamide (there is no reference to whether or not these treatments were stopped before administration of intravitreal dexamethazone implant), which might have played a role in fellow eye outcomes. In our patient (case 3) with bilateral ME secondary to RP who underwent fellow eye treatment 1 week after first eye administration of intravitreal dexamethazone implant [1], indeed, we did not experience the same bilateral effects as here reported by the authors. Similarly, we did not record bilateral effects of unilateral intravitreal dexamethazone implant in a recently published series of ME secondary to RVO [4].

In the current literature, there are several reports of bilateral effects of intravitreal treatments, particularly anti-VEGF drugs, unilaterally injected for different macular diseases. To the best of our knowledge, no similar data on bilateral effects of unilateral intravitreal dexamethazone implant are currently available in literature.

While it is difficult to generalize from a single case report, we agree with Drs Alhassan and Quintyn that it is worth assessing the effects of unilateral intravitreal dexamethazone implant before treating fellow eyes of RP patients with ME.

\section{References}

1. Srour M, Querques G, Leveziel N, Zerbib J, Tilleul J, BoulangerScemama E, Souied EH (2013) Intravitreal dexamethasone implant (Ozurdex) for macular edema secondary to retinitis pigmentosa. Graefes Arch Clin Exp Ophthalmol 251:1501-1506

2. Heckenlively JR, Jordan BL, Aptsiauri N (1999) Association of antiretinal antibodies and cystoid macular edema in patients with retinitis pigmentosa. Am J Ophthalmol 127:565-573

3. Yoshida N, Ikeda Y, Notomi S, Ishikawa K, Murakami Y, Hisatomi T, Enaida H, Ishibashi T (2013) Laboratory evidence of sustained chronic inflammatory reaction inretinitis pigmentosa. Ophthalmology 120:e5-e12

4. Querques L, Querques G, Lattanzio R, Gigante SR, Del Turco C, Corradetti G, Cascavilla ML, Bandello F (2013) Repeated intravitreal dexamethasone implant $\left(\right.$ Ozurdex $\left.{ }^{\circledR}\right)$ for retinal vein occlusion. Ophthalmologica 229:21-25
G. Querques · M. Srour • E. H. Souied

Department of Ophthalmology, Centre Hospitalier Intercommunal de Creteil University Paris Est Creteil, Creteil, France

G. Querques $(\square)$

Department of Ophthalmology, Centre Hospitalier Intercommunal de Creteil, 40 Avenue de Verdun, 94000 Creteil, France

e-mail: giuseppe.querques@hotmail.it 